\title{
Clinical, optical coherence tomography, and fundus autofluorescence findings in patients with intraocular tumors
}

This article was published in the following Dove Press journal:

Clinical Ophthalmology

7 October 2016

Number of times this article has been viewed

\section{Daniel Samuelsson \\ Monika Sznage \\ Karl Engelsberg \\ Elisabeth Wittström \\ Department of Ophthalmology, Lund University, Skane University Hospital, Lund, Sweden}

Purpose: To describe clinical, optical coherence tomography (OCT) and fundus autofluorescence (FAF) findings in patients with intraocular tumors and determine if OCT and FAF could be helpful in the differential diagnosis and management of different choroidal tumors.

Methods: Forty-nine patients with untreated, macular, midperipheral, and extrapapillary intraocular tumors were included. All patients underwent ophthalmic examination: best-corrected visual acuity, slit-lamp biomicroscopy, funduscopy, and standardized B mode, and if possible A mode, ultrasonography, and OCT and FAF imaging of the surface of the intraocular tumors.

Results: Of the 49 patients studied, 19 had choroidal nevi, ten had indeterminate choroidal melanocytic lesions (IMLs), ten had malignant melanomas, and ten had other choroidal tumors. The choroidal nevi revealed subretinal fluid (SRF) on OCT in only $11 \%$. FAF detected isoautofluorescence in $42 \%$, hypoautofluorescence in $37 \%$, patchy FAF pattern in $16 \%$, and a diffuse FAF pattern in 5\%. Seventy percent of patients with IML showed SRF on OCT and $20 \%$ showed tumor growth on follow-up, detected only by OCT and FAF imaging. FAF revealed a patchy pattern in $50 \%$ and a diffuse pattern in $40 \%$ of cases with IML. Ninety percent of the patients with choroidal melanoma had SRF on OCT and FAF revealed a patchy pattern in $60 \%$ and a diffuse pattern in $40 \%$. Patients with other choroidal tumors had SRF on OCT in $30 \%$ of cases and no characteristic pattern on FAF.

Conclusion: Both OCT and FAF were helpful in the differential diagnosis of choroidal nevi versus IMLs, choroidal melanomas, and other choroidal tumors. Also, detailed and periodical clinical evaluation of patients with intraocular tumors using OCT and FAF imaging for the detection of both SRF and FAF patterns overlying the tumor can be useful for detection of tumor growth.

Keywords: intraocular tumors, optical coherence tomography, fundus autofluorescence, tumor growth

\section{Introduction}

During the past 2 decades, two noninvasive imaging methods, optical coherence tomography (OCT) and fundus autofluorescence (FAF) photography, have become available in routine clinical practice. These methods provide qualitative and quantitative information about retinal changes that are not only important for the management of patients with many retinal diseases but also for patients with intraocular tumors. ${ }^{1-3}$ OCT and FAF imaging, when used together and combined with fundus photography, fluorescein angiography/indocyanine green angiography (FA/ICG), and ultrasonography, have been shown to be useful for early tumor diagnosis, monitoring of tumor growth, and follow-up of the results of therapies. ${ }^{1-11}$
Correspondence: Elisabeth Wittström Department of Ophthalmology, Lund University, SE-22I 85 Lund, Sweden Tel +4646I7 6849

Fax +4646176141

Email elisabeth.wittstrom@skane.se (c) (i) () 2016 Samuelsson et al. This work is published and licensed by Dove Medical Press Limited. The full terms of this license are available at https://www.dovepress.com/terms.php (c) ${ }_{\mathrm{BY}} \mathrm{NC}$ and incorporate the Creative Commons Attribution - Non Commercial (unported, v3.0) License (http://creativecommons.org/licenses/by-nc/3.0/). By accessing the work you hereby accept the Terms. Non-commercial uses of the work are permitted without any further permission from Dove Medical Press Limited, provided the work is properly attributed. For permission for commercial use of this work, please see paragraphs 4.2 and 5 of our Terms (https://www.dovepress.com/terms.php). 
Today, spectral domain (SD)-OCT can provide crosssectional images of the individual retinal layers and SD-OCT scans have been used for the detection and quantification of secondary retinal, retinal pigment epithelium (RPE), and choroidal changes overlying ocular tumors, for example, subretinal fluid (SRF), which may have predictive value for ocular tumor growth and could be a clinical indicator for malignancy in eyes with indeterminate choroidal melanocytic lesions (IMLs). ${ }^{1,6,10-12}$ OCT was found to be more sensitive than clinical examination or ultrasonography for the identification of SRF. ${ }^{13}$ SD-OCT findings have recently been reported to be helpful in the diagnosis, follow-up, and treatment of different choroidal tumors, such as nevi, melanomas, metastases, hemangiomas, and lymphomas. ${ }^{6,14-18}$

Secondary retinal and RPE changes associated with various intraocular tumors can also be documented by FAF. FAF imaging is a noninvasive method that provides a topographical map of lipofuscin, which is the main fundus fluorophore ${ }^{19}$ Lipofuscin (a mixture of proteins, lipids, and small chromophores) is the end product of phagocytosed photoreceptor outer segments, mostly accumulating within the RPE cells. It has been shown that excessive accumulation of lipofuscin may impair the function of RPE cells, which may cause atrophy or death of the cells and, secondarily, the death of the overlying photoreceptors. ${ }^{20,21}$ Using FAF, it is possible to assess the metabolic health and functionality of the RPE cells. ${ }^{20}$

Today, FAF can be measured using a confocal scanning laser ophthalmoscope. FAF images can also be obtained using a Topcon fundus-based camera system. The autofluorescence images produced by a confocal scanning laser ophthalmoscope and fundus camera are similar., ${ }^{2,3}$ Several authors have used confocal scanning laser ophthalmoscope to examine FAF images in patients with intraocular tumors, ${ }^{8-10,22-25}$ while others have studied the autofluorescence using a fundus camera. ${ }^{9,26-31}$

The documented growth of an IML has been used as a surrogate for malignancy or a risk factor for the malignancy of the lesion since 1994. ${ }^{32-35}$ There is evidence that large tumors are associated with a poor prognosis and higher metastatic rates. ${ }^{36-38}$ Shields et al ${ }^{37}$ reported that each extra millimeter in melanoma thickness meant a 5\% higher risk of metastasis. Recently, Damato et al ${ }^{38}$ revealed that ocular treatment of choroidal melanoma may prevent tumor growth, dedifferentiation, and metastatic disease in younger patients with small and less malignant tumors. The findings in these two reports illustrate the importance of early detection and treatment of malignant uveal melanomas to ensure a more favorable prognosis. Apart from clinical risk factors, such as large tumor size, documented tumor growth, the presence of chromosome 3 monosomy (loss of heterozygosity), and somatic mutations in genes, GNAQ or GNA11 have also been found to be strongly associated with death due to metastases in patients with uveal melanoma. ${ }^{39-42}$

A good clinical evaluation of intraocular tumors, especially, of IML should include examination with ultrasound, OCT, FAF, FA/ICG and, if necessary, biopsy for histological, cytological, and genetic analysis to enhance the accuracy of early diagnosis and improve prognosis. ${ }^{35}$

In this study, we have evaluated the clinical, OCT, and FAF findings in 49 patients with intraocular tumors to determine if OCT and FAF could be helpful in the differential diagnosis and management of different choroidal tumors.

\section{Subjects and methods}

Forty-nine patients with untreated, macular, midperipheral, and juxtapapillary intraocular tumors, who had been periodically examined or who were referred for examination due to the finding of a new intraocular tumor between 2014 and 2015, were prospectively included in the study and follow-up between 5 and 23 months. Patients were excluded if they had a choroidal tumor that had been treated previously, had nonclear media that would reduce the quality of the OCT scans and FAF images, or had very peripheral choroidal tumors that could not be imaged using OCT and FAF.

A diagnostic decision was made by an experienced specialist in ocular oncology for each patient included in this study, on the basis of clinical manifestation, including A- and B-scan ultrasonography, biomicroscopy, fundus photography, and in some cases FA/ICG characteristics. The characteristics showed in OCT and FAF under study were not used to confirm the initial diagnosis. In one patient, the choroidal melanoma was diagnosed by transretinal biopsy. The patients studied were divided into four groups: nevi, IMLs, melanomas, and other choroidal tumors, which were compared. All patients included in the study underwent ophthalmic examination, including best-corrected visual acuity (VA), slit-lamp biomicroscopy, funduscopy, standardized B mode, and if possible A mode, ultrasonography, and color fundus photography. Some patients also underwent FA and ICG. In addition, all patients underwent OCT and FAF imaging of the surface of intraocular tumors and macula.

A- and B-scan ophthalmic ultrasonography was performed on all patients using a CineScan S (Quantel Medical, Clermont-Ferrand, France).

OCT was performed using the SD 3D OCT-1000, version 3.00 software (Topcon, Tokyo, Japan). The 3D macular scan option was used for all scans in this study, centered on 
the tumor and the fovea, covering $6 \times 6 \mathrm{~mm}$, with a resolution of $512 \times 128$ pixels. The presence of SRF was documented in each studied group and compared between groups.

FAF was performed with a Topcon TRC-50DX retinal camera (Topcon, with an excitation bandpass filter of $535-585 \mathrm{~nm}$ and a barrier bandpass filter of $605-715 \mathrm{~nm}$ ). All OCT and FAF images were reviewed by two reviewers. The FAF patterns in choroidal tumors were classified as patchy or diffuse when applicable. The patchy pattern was defined as the presence of distinct areas of increased FAF between areas of normal autofluorescence. The diffuse pattern was characterized by the presence of increased FAF with indistinct borders over a larger part of the tumor in the absence of intervening areas of normal FAF. ${ }^{8}$ Abnormal autofluorescence was also defined as either increased or decreased FAF in comparison with background FAF, and classified as isoautofluorescence, hypoautofluorescence, or a combination of hypoauto and hyperautofluorescence (mixed). ${ }^{23,27}$

The following patient characteristics and clinical examination findings at the time of OCT and FAF imaging were recorded and analyzed: sex (male, female), age, affected eye (right eye, left eye), initial Snellen VA, tumor location (distance to the optic nerve and to the foveola $[\mathrm{mm}]$ ) and largest basal diameter ( $\mathrm{mm}$ ) measured using fundus photography, tumor thickness (mm) measured by B-scan ultrasonography, tumor color (pigmented, partially pigmented, and nonpigmented), tumor configuration (flat, dome-shaped), SRF on OCT (yes/no), and FAF findings (patchy, diffuse, isoautofluorescence, hypoautofluorescence, or a combination of hypoauto and hyperautofluorescence [mixed]).

The study was approved by the Ethics Committee of Lund University and all the participants gave their written consent to participate according to the principles outlined in the Universal Declaration of Helsinki.

\section{Statistical analysis}

The data were analyzed using computer software SPSS version 22 (IBM Corporation, Armonk, NY, USA). VA was measured using a Snellen chart and then converted to the logarithm of the minimum angle of resolution ( $\log M A R)$ before statistical analysis. The Mann-Whitney $U$-test was used for continuous variables and Fisher's exact test was used for categorical variables. Values of $P \leq 0.05$ were considered to indicate statistical significance.

\section{Results}

The study consisted of 49 patients with intraocular tumors (19 males and 30 females). The mean age was 64 years (median, 68 years; range, 18-93 years). The intraocular tumors were in the right eye in 24 patients and in the left eye in 25 patients. The mean VA ( $\log$ MAR) was 0.26 (median, 0.16; range, 0.0-2.0). The mean distance from the tumor margin to the optic nerve was $2.6 \mathrm{~mm}$ (median, $2.1 \mathrm{~mm}$; range, 0.0-9.3 $\mathrm{mm}$ ) and the mean distance to the foveola was $1.7 \mathrm{~mm}$ (median, 0.8; range, $0.0-7.5 \mathrm{~mm}$ ). The mean tumor thickness was $2.3 \mathrm{~mm}$ (median, $2.4 \mathrm{~mm}$; range, $0.0-11 \mathrm{~mm}$ ) and the mean value of the larger basal diameter of the tumor was $6.4 \mathrm{~mm}$ (median, $6.0 \mathrm{~mm}$; range, 1.6-14.5 $\mathrm{mm}$ ). Of the 49 patients studied, 19 had choroidal nevi, ten had IMLs, ten had malignant choroidal melanomas, and ten had other choroidal tumors (two choroidal metastases, three choroidal osteomas, and five choroidal hemangiomas) (Table 1). The mean follow-up (months) for all tumors was 13.5 \pm 5.4 ; choroidal nevi, 14.8 \pm 4.7 ; IMLs, $16.9 \pm 4.8$; choroidal melanomas, 10.4 \pm 5.5 ; and other choroidal tumors, 10.7 \pm 4.8 . Both patchy and diffuse FAF patterns were found to be associated with decreased VA in all studied tumor groups (Tables 1 and 2).

\section{Patients with choroidal nevi under observation}

Nineteen of the patients had a diagnosis of choroidal nevus and had been managed by regular observation. The characteristics, clinical, OCT, and FAF findings, are given in Tables 1 and 3. Fundus photographs, FAF images, and OCT scans of two patients with choroidal nevi are presented in Figure 1.

No significant difference was observed between patients with choroidal nevi and IMLs, melanomas, and other choroidal tumors regarding sex, age, affected eye, and distance from the tumor margin to the optic nerve and foveola (Table 1). Patients with choroidal nevi had significantly better VA $(\log M A R)$ than those with IMLs and melanomas ( $P=0.016$ and $P=0.040$, respectively) (Table 1 ).

Significant differences were observed between choroidal nevus and IMLs, choroidal melanomas, and other choroidal tumors regarding tumor thickness, largest basal diameter, tumor color, configuration, and fluid on OCT and FAF findings (Tables 1 and 3). Choroidal nevi were often pigmented (89\%), had a flat configuration $(79 \%)$, had no fluid on OCT (89\%), and often demonstrated isoautofluorescence (42\%) and hypoautofluorescence $(37 \%)$ on FAF imaging (Table 3).

\section{Patients with indeterminate choroidal melanocytic lesion under observation}

Ten of the patients had a diagnosis of IML and had been managed by regular observation. The characteristics, clinical, OCT, and FAF findings, are given in Tables 1-4. 
Table I Demographics and clinical findings in patients with nevi, indeterminate choroidal melanocytic lesions, choroidal melanomas, and other choroidal tumors, together with statistical comparison

\begin{tabular}{|c|c|c|c|c|c|}
\hline & All tumors $n=49$ & Nevi $n=19$ & IMLs $n=10$ & $\begin{array}{l}\text { Melanomas } \\
n=10\end{array}$ & $\begin{array}{l}\text { Other choroida } \\
\text { tumors } n=10\end{array}$ \\
\hline \multicolumn{6}{|l|}{ Sex } \\
\hline Male & $19(38 \%)$ & $8(42 \%)$ & $6(60 \%)$ & $2(20 \%)$ & $3(30 \%)$ \\
\hline Female & $30(62 \%)$ & II (58\%) & $4(40 \%)$ & $8(80 \%)$ & $7(70 \%)$ \\
\hline$P$-value & & & 0.450 & 0.414 & 0.694 \\
\hline \multicolumn{6}{|l|}{ Age (years) } \\
\hline Mean \pm SD & $64 \pm 15$ & $68 \pm 9$ & $65 \pm 10$ & $69 \pm 15$ & $51 \pm 22$ \\
\hline Median (min-max) & $68(18-93)$ & $69(52-90)$ & $64(53-85)$ & 70 (44-93) & $55(18-74)$ \\
\hline$P$-value & & & 0.377 & 0.701 & 0.094 \\
\hline \multicolumn{6}{|l|}{ Affected eye } \\
\hline OD & 24 (49\%) & II (58\%) & $5(50 \%)$ & $5(50 \%)$ & $3(30 \%)$ \\
\hline OS & $25(51 \%)$ & $8(42 \%)$ & $5(50 \%)$ & $5(50 \%)$ & $7(70 \%)$ \\
\hline$P$-value & & & 0.714 & 0.714 & 0.245 \\
\hline \multicolumn{6}{|c|}{ Visual acuity (logMAR) } \\
\hline Mean \pm SD & $0.26 \pm 0.40$ & $0.10 \pm 0.11$ & $0.31 \pm 0.26$ & $0.49 \pm 0.62$ & $0.30 \pm 0.40$ \\
\hline Median (min-max) & $0.16(0.0-2.0)$ & $0.10(0.0-0.40)$ & $0.18(0.0-0.7)$ & $0.30(0.0-2.0)$ & $0.15(0.0-1.22)$ \\
\hline$P$-value & & & $0.016 *$ & $0.040^{*}$ & 0.308 \\
\hline \multicolumn{6}{|c|}{ Tumor location distance to optic nerve $(\mathrm{mm})$} \\
\hline Mean \pm SD & $2.6 \pm 2.4$ & $2.7 \pm 2.6$ & $3.1 \pm 2.5$ & $2.8 \pm 2.2$ & $1.9 \pm 2.5$ \\
\hline Median (min-max) & $2.1(0.0-9.3)$ & $2.2(0.0-9.3)$ & $3.2(0.0-7.5)$ & $2.6(0.0-6.7)$ & I.I (0.0-7.8) \\
\hline$P$-value & & & 0.573 & 0.804 & 0.286 \\
\hline \multicolumn{6}{|c|}{ Distance to foveola (mm) } \\
\hline Mean \pm SD & $1.7 \pm 2.1$ & $2.0 \pm 2.1$ & $1.6 \pm 2.8$ & $1.5 \pm 1.9$ & $1.6 \pm 2.4$ \\
\hline Median (min-max) & $0.8(0.0-7.5)$ & $1.3(0.0-6.0)$ & $0.6(0.0-7.5)$ & $0.5(0.0-5.4)$ & $0.0(0.0-6.0)$ \\
\hline$P$-value & & & 0.429 & 0.429 & 0.429 \\
\hline \multicolumn{6}{|c|}{ Tumor thickness (mm) } \\
\hline Mean \pm SD & $2.3 \pm 1.7$ & $1.3 \pm 1.2$ & $2.6 \pm 0.5$ & $3.6 \pm 2.6$ & $2.6 \pm 1.4$ \\
\hline Median (min-max) & $2.4(0.0-11.0)$ & $1.8(0.0-3.2)$ & $2.4(1.9-3.3)$ & $3.1(1.9-11.0)$ & $2.7(1.0-4.6)$ \\
\hline$P$-value & & & $0.005^{*}$ & $<0.00 I^{*}$ & $0.027^{*}$ \\
\hline \multicolumn{6}{|c|}{ Largest basal diameter of tumor $(\mathrm{mm})$} \\
\hline Mean \pm SD & $6.4 \pm 3.2$ & $4.1 \pm 1.6$ & $7.5 \pm 3.3$ & $8.6 \pm 3.1$ & $7.4 \pm 3.3$ \\
\hline Median (min-max) & $6.0(1.6-14.5)$ & $3.8(2.1-8.5)$ & $8.3(1.6-11.4)$ & $8.6(3.7-13.3)$ & $7.0(3.1-14.5)$ \\
\hline$P$-value & & & $0.008^{*}$ & $<0.00 I^{*}$ & $0.003^{*}$ \\
\hline \multicolumn{6}{|c|}{ Follow-up (months) } \\
\hline Mean \pm SD & $13.5 \pm 5.4$ & $14.8 \pm 4.7$ & $16.9 \pm 4.8$ & $10.4 \pm 5.5$ & $10.7 \pm 4.8$ \\
\hline Median (min-max) & $14.0(5-23)$ & $15.0(6-23)$ & $17.5(7-22)$ & $7.5(6-20)$ & $10.5(5-19)$ \\
\hline$P$-value & & & 0.247 & $0.045^{*}$ & $0.040^{*}$ \\
\hline
\end{tabular}

Notes: $P$-value - nevus vs IML, melanoma, and other choroidal tumors. * denotes statistical significance.

Abbreviations: IML, indeterminate choroidal melanocytic lesion; logMAR, logarithm of the minimum angle of resolution; OD, right eye; OS, left eye; SD, standard deviation.

Fundus photographs, FAF images, and OCT scans of two patients with IML are presented in Figure 1.

No significant difference was observed between patients with IML and patients with choroidal melanoma and other choroidal tumors regarding sex, age, affected eye, VA ( $\log M A R)$, distance from the tumor margin to the optic nerve and foveola, tumor thickness, largest basal diameter, and fluid on OCT and FAF findings (Tables 2 and 4).

A significant difference was observed only between IML and other choroidal tumors regarding tumor color $(P=0.001)$ (Table 4). Two of ten (20\%) patients with IML exhibited tumor growth on follow-up $<12$ months. This was detected only with OCT and FAF imaging. Color fundus photographs, FAF images, and OCT scans before and 11 months after presentation of one patient with IML who showed tumor growth and FAF pattern changes from patchy to diffuse are shown in Figure 1.

\section{Patients with choroidal melanoma}

Ten of the patients had a diagnosis of choroidal melanoma. The characteristics, clinical, OCT, and FAF findings, are given in Tables 1-4. Color fundus photographs, FAF images, and OCT scans of two patients with melanoma are presented in Figure 2. No significant difference was observed between 
Table 2 Statistical comparison of demographics and clinical findings in patients with indeterminate choroidal melanocytic lesions, choroidal melanomas, and other choroidal tumors

\begin{tabular}{|c|c|c|c|c|c|c|}
\hline & IMLs $\mathbf{n}=10$ & Melanomas $n=10$ & $P$-value ${ }^{a}$ & $\begin{array}{l}\text { Other choroidal } \\
\text { tumors } n=10\end{array}$ & $P$-value ${ }^{a}$ & $P$-value \\
\hline \multicolumn{7}{|l|}{ Sex } \\
\hline Male & $6(60 \%)$ & $2(20 \%)$ & & $3(30 \%)$ & & \\
\hline Female & $4(40 \%)$ & $8(80 \%)$ & 0.170 & $7(70 \%)$ & 0.370 & 0.635 \\
\hline \multicolumn{7}{|l|}{ Age (years) } \\
\hline Mean \pm SD & $65 \pm 10$ & $69 \pm 15$ & & $5 I \pm 22$ & & \\
\hline Median (min-max) & $64(53-85)$ & $70(44-93)$ & 0.393 & $55(18-74)$ & 0.393 & 0.075 \\
\hline \multicolumn{7}{|l|}{ Affected eye } \\
\hline OD & $5(50 \%)$ & $5(50 \%)$ & & $3(30 \%)$ & & \\
\hline OS & $5(50 \%)$ & $5(50 \%)$ & 1.000 & $7(70 \%)$ & 0.650 & 0.650 \\
\hline \multicolumn{7}{|c|}{ Visual acuity (logMAR) } \\
\hline Mean \pm SD & $0.3 \mathrm{I} \pm 0.26$ & $0.49 \pm 0.62$ & & $0.30 \pm 0.40$ & & \\
\hline Median (min-max) & $0.18(0.0-0.7)$ & $0.30(0.0-2.0)$ & 0.729 & $0.15(0.0-1.22)$ & 0.529 & 0.481 \\
\hline \multicolumn{7}{|c|}{ Tumor location distance to optic nerve $(\mathrm{mm})$} \\
\hline Mean \pm SD & $3.1 \pm 2.5$ & $2.8 \pm 2.2$ & & $1.9 \pm 2.5$ & & \\
\hline Median (min-max) & $3.2(0.0-7.5)$ & $2.6(0.0-6.7)$ & 0.853 & I.I (0.0-7.8) & 0.280 & 0.218 \\
\hline \multicolumn{7}{|c|}{ Distance to foveola $(\mathrm{mm})$} \\
\hline Mean \pm SD & $1.6 \pm 2.8$ & $1.5 \pm 1.9$ & & $1.6 \pm 2.4$ & & \\
\hline Median (min-max) & $0.6(0.0-7.5)$ & $0.5(0.0-5.4)$ & 1.000 & $0.0(0.0-6.0)$ & 0.796 & 0.853 \\
\hline \multicolumn{7}{|c|}{ Tumor thickness $(\mathrm{mm})$} \\
\hline Mean \pm SD & $2.6 \pm 0.5$ & $3.6 \pm 2.6$ & & $2.6 \pm 1.4$ & & \\
\hline Median (min-max) & $2.4(1.9-3.3)$ & $3.1(1.9-11.0)$ & 0.280 & $2.7(1.0-4.6)$ & 1.000 & 0.579 \\
\hline \multicolumn{7}{|c|}{ Largest basal diameter of tumor ( $\mathrm{mm})$} \\
\hline Mean \pm SD & $7.5 \pm 3.3$ & $8.6 \pm 3.1$ & & $7.4 \pm 3.3$ & & \\
\hline Median (min-max) & $8.3(1.6-11.4)$ & $8.6(3.7-13.3)$ & 0.481 & $7.0(3.1-14.5)$ & 0.739 & 0.353 \\
\hline \multicolumn{7}{|l|}{ Follow-up } \\
\hline Mean \pm SD & $16.9 \pm 4.8$ & $10.4 \pm 5.5$ & & $10.7 \pm 4.8$ & & \\
\hline Median (min-max) & $17.5(7-22)$ & $7.5(6-20)$ & $0.011^{*}$ & $10.5(5-19)$ & $0.015^{*}$ & 0.853 \\
\hline
\end{tabular}

Notes: a ${ }^{P}$-value, IML vs melanoma and other choroidal tumors; ${ }^{b}$-value, melanoma vs other choroidal tumors. * denotes statistical significance.

Abbreviations: IML, indeterminate choroidal melanocytic lesion; logMAR, logarithm of the minimum angle of resolution; OD, right eye; OS, left eye; SD, standard deviation.

Table 3 Statistical comparison of clinical findings of nevi versus indeterminate choroidal melanocytic lesions, choroidal melanomas, and other choroidal tumors

\begin{tabular}{|c|c|c|c|c|c|c|c|}
\hline & Nevi $n=19(\%)$ & IMLs $\mathrm{n}=10$ (\%) & $P$-value & $\begin{array}{l}\text { Melanomas } \\
n=10(\%)\end{array}$ & $P$-value & $\begin{array}{l}\text { Other choroidal } \\
\text { tumors } n=10(\%)\end{array}$ & $P$-value \\
\hline \multicolumn{8}{|l|}{ Tumor color } \\
\hline Pigmented & $17(89)$ & $3(30)$ & & $2(20)$ & & $0(0)$ & \\
\hline Partially pigmented & $2(11)$ & $5(50)$ & & $4(40)$ & & $0(0)$ & \\
\hline Nonpigmented & $0(0)$ & $2(20)$ & $0.002 *$ & $4(40)$ & $<0.00 I^{*}$ & $10(100)$ & $<0.001 *$ \\
\hline \multicolumn{8}{|l|}{ Tumor configuration } \\
\hline Flat & $15(79)$ & $0(0)$ & & $0(0)$ & & $0(0)$ & \\
\hline Dome-shaped & $4(21)$ & $10(100)$ & $<0.00 I^{*}$ & $10(100)$ & $<0.001 *$ & $10(100)$ & $<0.00 I^{*}$ \\
\hline \multicolumn{8}{|l|}{ Fluid on OCT } \\
\hline Yes (SRF) & $2(I I)$ & $7(70)$ & & $9(90)$ & & $3(30)$ & \\
\hline No (SRF) & $17(89)$ & $3(30)$ & $0.002 *$ & $I(10)$ & $<0.00 I^{*}$ & $7(70)$ & 0.306 \\
\hline \multicolumn{8}{|l|}{ FAF findings } \\
\hline Patchy & $3(16)$ & $5(50)$ & & $6(60)$ & & $2(20)$ & \\
\hline Diffuse & $\mathrm{I}(5)$ & $4(40)$ & & $4(40)$ & & $I(10)$ & \\
\hline Isoauto & $8(42)$ & $\mathrm{I}(\mathrm{I0})$ & & $0(0)$ & & $4(40)$ & \\
\hline Hypoauto & $7(37)$ & $0(0)$ & & $0(0)$ & & $\mathrm{I}(\mathrm{I0})$ & \\
\hline Hypoauto/hyperauto (mixed) & $0(0)$ & $0(0)$ & $<0.00 I^{*}$ & $0(0)$ & $<0.00 I^{*}$ & $2(20)$ & $<0.00 I^{*}$ \\
\hline \multicolumn{8}{|l|}{ FAF findings and VA } \\
\hline Patchy $(V A<20 / 20)$ & $2 / 3(67)$ & $5 / 5(100)$ & & $4 / 6(67)$ & & $1 / 2(50)$ & \\
\hline Diffuse (VA <20/20) & $0 / 1(0)$ & $3 / 4(75)$ & & $4 / 4(100)$ & & $\mathrm{I} / \mathrm{I}(100)$ & \\
\hline
\end{tabular}

Note: * denotes statistical significance.

Abbreviations: FAF, fundus autofluorescence; IML, indeterminate choroidal melanocytic lesion; OCT, optical coherence tomography; SRF, subretinal fluid; VA, visual acuity. 

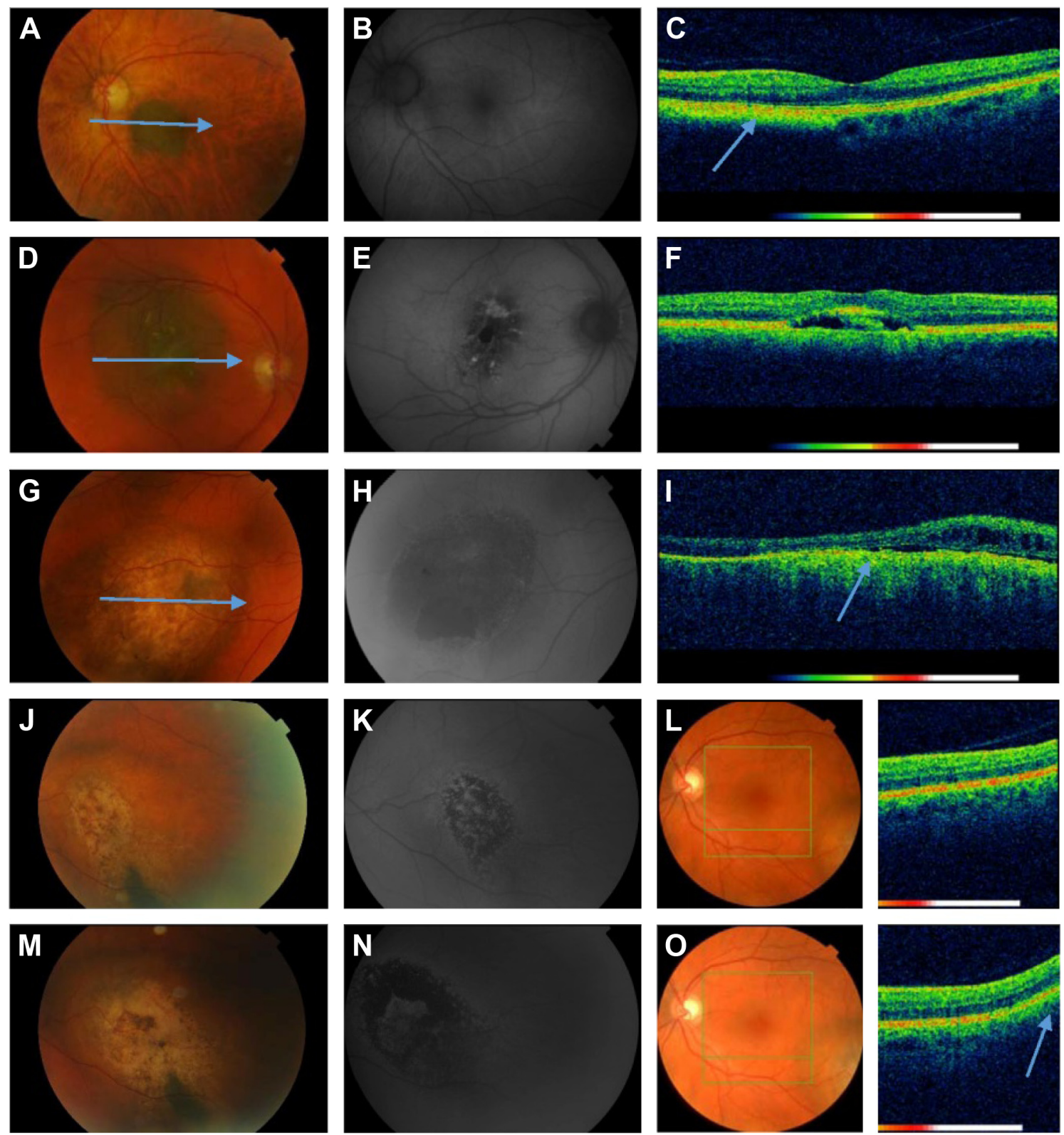

Figure I Color fundus photographs, FAF images, and OCT scans of patients with choroidal nevi and indeterminate choroidal melanocytic lesions.

Notes: Images from a female aged 73 with a choroidal nevus in her left eye (A-C). (A) Color fundus photo shows pigmented choroidal nevus in the macula. (B) FAF image shows only isoautofluorescence over the nevus. (C) OCT scan of the surface of the nevus reveals a bright signal (arrow) from the nevus underlying the RPE and no evidence of intra- or subretinal fluid. Images from a male aged 75 with choroidal nevus in his right eye, unchanged over the past year (D-F). (D) Color fundus photo shows partially pigmented choroidal nevus in the macula of the right eye. (E) FAF image shows a patchy pattern characterized by distinct areas of increased autofluorescence between areas of normal autofluorescence. (F) OCT scan of the surface of the nevus shows irregularity at the level of the RPE and minimal subretinal fluid. Images from a male aged 63 with an IML in his right eye, unchanged over the past 4 years $(\mathbf{G}-\mathbf{I})$. (G) Color fundus photo shows a partially pigmented IML in the macula. (H) FAF image shows a patchy pattern. (I) OCT scan of the surface of the IML reveals the elevated choroidal mass (arrow) with RPE changes and intraretinal fluid. Images from a female aged 53 with an IML in her left eye show tumor growth and FAF pattern changes from patchy to diffuse II months after presentation (J-O). (J) Color fundus photo shows a partially pigmented IML. (K) FAF image shows a patchy pattern. (L) Color fundus photo and OCT scan reveal IML temporal. (M) Color fundus photo shows more pigmented IML. (N) FAF image shows a diffuse pattern II months after presentation. (O) Color fundus photo and OCT scan show tumor growth to the macula center (arrow). The arrows at the color fundus photographs (A, D, G) correspond to the B-scan lines of the OCT images.

Abbreviations: FAF, fundus autofluorescence; IML, indeterminate choroidal melanocytic lesion; OCT, optical coherence tomography; RPE, retinal pigment epithelium.

patients with choroidal melanoma and IML and other choroidal tumors regarding sex, age, affected eye, distance from the tumor margin to the optic nerve and foveola, tumor thickness, or largest basal diameter (Table 2). Significant differences were observed between patients with choroidal melanoma and other choroidal tumors regarding tumor color and fluid on OCT ( $P=0.011$ and $P=0.020$, respectively) (Table 4). One patient with a tumor thickness of $1.9 \mathrm{~mm}$, which was nonpigmented, was diagnosed as having choroidal melanoma by transretinal biopsy (Figure 2). 
Table 4 Statistical comparison of clinical findings of indeterminate choroidal melanocytic lesions versus choroidal melanomas, other choroidal tumors, and choroidal melanomas versus other choroidal tumors

\begin{tabular}{|c|c|c|c|c|c|c|}
\hline & IMLs n=I 0 (\%) & $\begin{array}{l}\text { Melanomas } \\
n=10(\%)\end{array}$ & $P$-value ${ }^{a}$ & $\begin{array}{l}\text { Other choroidal } \\
\text { tumors } n=10(\%)\end{array}$ & $P$-value ${ }^{a}$ & $P$-value ${ }^{b}$ \\
\hline \multicolumn{7}{|l|}{ Tumor color } \\
\hline Pigmented & $3(30)$ & $2(20)$ & & $0(0)$ & & \\
\hline Partially pigmented & $5(50)$ & $4(40)$ & & $0(0)$ & & \\
\hline Nonpigmented & $2(20)$ & $4(40)$ & 0.727 & $10(100)$ & $0.00 I^{*}$ & $0.011 *$ \\
\hline \multicolumn{7}{|l|}{ Tumor configuration } \\
\hline Flat & $0(0)$ & $0(0)$ & & $0(0)$ & & \\
\hline Dome-shaped & $10(100)$ & $10(100)$ & & $10(100)$ & & \\
\hline \multicolumn{7}{|l|}{ Fluid on OCT } \\
\hline Yes (SRF) & $7(70)$ & $9(90)$ & & $3(30)$ & & \\
\hline No (SRF) & $3(30)$ & $I(10)$ & 0.582 & $7(70)$ & 0.179 & $0.020^{*}$ \\
\hline \multicolumn{7}{|l|}{ FAF findings } \\
\hline Patchy & $5(50)$ & $6(60)$ & & $2(20)$ & & \\
\hline Diffuse & $4(40)$ & $4(40)$ & & $\mathrm{I}(\mathrm{I0})$ & & \\
\hline Isoauto & $I(10)$ & $0(0)$ & & $4(40)$ & & \\
\hline Hypoauto & $0(0)$ & $0(0)$ & & $\mathrm{I}(10)$ & & \\
\hline Hypoauto/hyperauto (mixed) & $0(0)$ & $0(0)$ & 1.000 & $2(20)$ & 0.230 & 0.141 \\
\hline
\end{tabular}

Notes: a ${ }^{P}$-value, IMLs vs choroidal melanomas and other choroidal tumors; ${ }^{b}$-value, melanomas vs other choroidal tumors. * denotes statistical significance.

Abbreviations: FAF, fundus autofluorescence; IML, indeterminate choroidal melanocytic lesion; OCT, optical coherence tomography; SRF, subretinal fluid.

\section{Patients with other choroidal tumors}

Ten of the patients had been classified as having other choroidal tumors. Five of them had choroidal hemangiomas, three osteomas, and two metastases (one breast cancer and one systemic lymphoma). The characteristics, clinical, OCT, and FAF findings, are given in Tables 1-4. Color fundus photographs, FAF images, and OCT scans of four patients with other choroidal tumors are presented in Figures 3 and 4.

There was no significant difference between other choroidal tumors and IML and choroidal melanoma regarding sex, age, affected eye, VA, distance from tumor margin to the optic nerve and foveola, tumor thickness, or largest basal diameter (Table 2). All other choroidal tumors were nonpigmented; $70 \%$ of them had no fluid on OCT images and $40 \%$ showed isoautofluorescence on FAF images (Table 4).

\section{Discussion}

The results of the present study demonstrate that the choroidal nevi located in the macular region, midperiphery, and juxtapapillary regions were pigmented in $89 \%$, flat in $79 \%$, and only $11 \%$ showed SRF on OCT. These findings can be compared with those of several other studies on choroidal nevi using OCT imaging in which it was reported that between $16 \%$ and $30 \%$ of the patients with choroidal nevi had SRF on OCT. ${ }^{7,13,14}$ The choroidal nevi included in the present study exhibited isoautofluorescence in $42 \%$, hypoautofluorescence in $37 \%$, a patchy FAF pattern in $16 \%$, and a diffuse pattern in $5 \%$ of the cases. The FAF abnormalities in the choroidal nevi identified in our study were somewhat different from FAF findings reported by Shields et $\mathrm{al}^{26}$ who found hypoautofluorescence in $56 \%$ and isoautofluorescence in only $18 \%$ of cases with choroidal nevi. The higher rate of isoautofluorescence in our study could be due to the fact that maybe we included less chronic choroidal nevi with less chronic RPE degenerative features compared to Shields et al. ${ }^{26}$ We also found both patchy FAF patterns and diffuse patterns in choroidal nevi, while Gündüz et al ${ }^{8}$ reported that all the choroidal nevi in their study showed a patchy FAF pattern. The present study demonstrates that apart from tumor features, such as tumor thickness, and the largest basal diameter, OCT and FAF findings were also very valuable in differentiating choroidal nevi from IMLs and choroidal melanomas.

The IMLs in this study exhibited significantly greater tumor thickness and larger basal diameter than the choroidal nevi, and did not show any significant differences between choroidal melanoma and other choroidal tumors regarding VA, all tumor characteristics, or OCT and FAF findings. Singh et $\mathrm{a}^{10}$ studied 38 patients with IML or with a small tumor according to the size-based nomenclature used in the Collaborative Ocular Melanoma Study using SD-OCT and FAF imaging and found that nearly $60 \%$ of cases had SRF on OCT. Espinoza et $\mathrm{al}^{4}$ detected SRF in $63 \%$ of cases of IML. SRF was detected in $70 \%$ of the cases of IML using OCT in this study, which is very similar to the OCT findings reported in the two studies mentioned above. Patients with IMLs had 

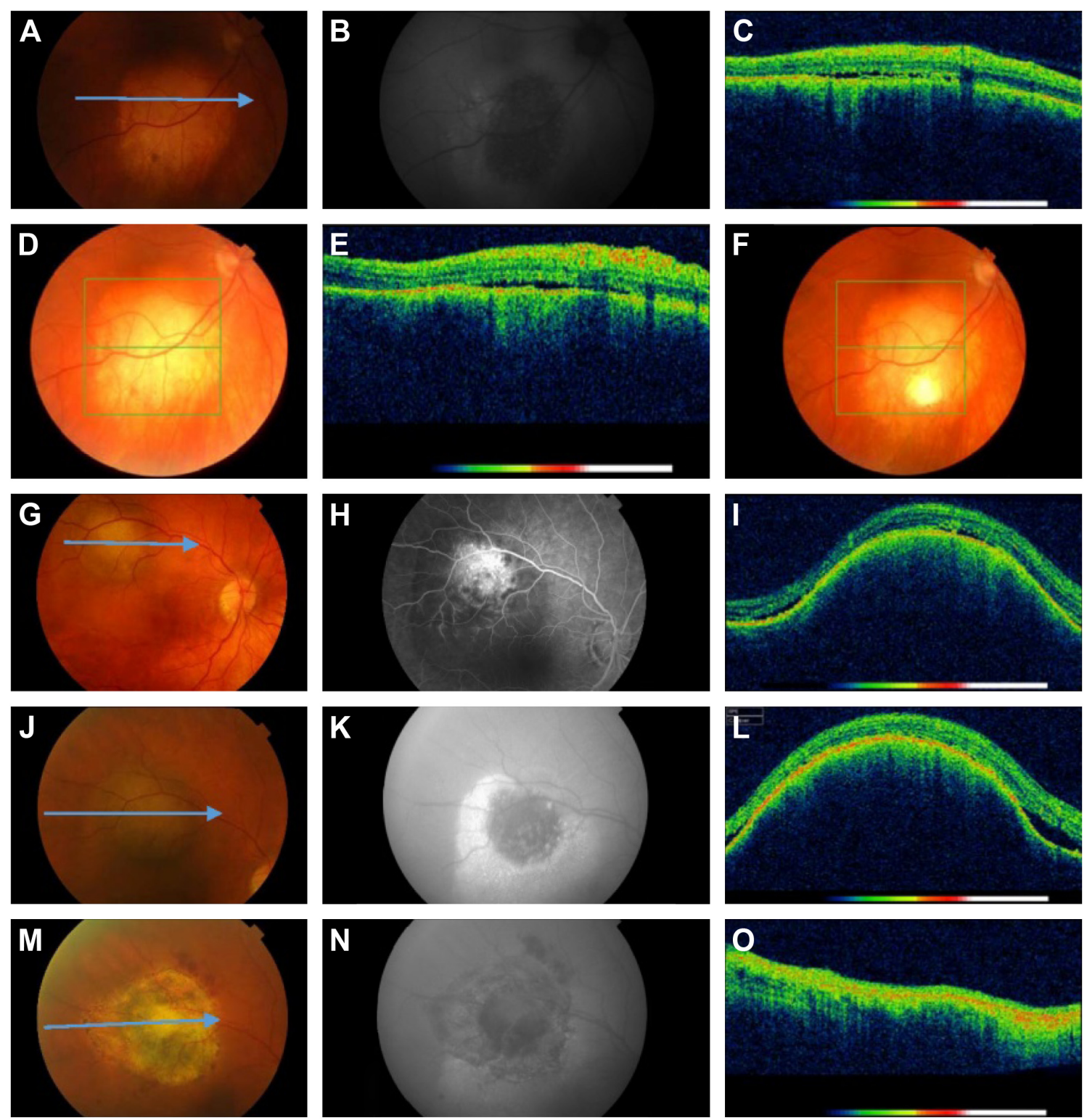

Figure 2 Color fundus photographs, FAF images, and OCT scans of patients with melanomas.

Notes: Images from a female aged 45 with a nonpigmented tumor in her right eye, confirmed as a malignant melanoma by transretinal biopsy (A-F). (A) Color fundus photo shows nonpigmented tumor. (B) FAF image shows a patchy pattern characterized by distinct areas of increased autofluorescence between areas of normal autofluorescence. (C and E) OCT scans before (C) and after (E) transretinal biopsy are unchanged with minimal subretinal fluid. (D and $\mathbf{F})$ Color fundus photos before (D) and after (F) biopsy show no complications. Images from a female aged 63 with a partially pigmented tumor in her right eye confirmed as choroidal melanoma by documented tumor growth (G-O). (G) Color fundus photo shows partially pigmented melanoma. (J) Tumor growth. (M) marked tumor reduction 8 months after thermotherapy. (H) Fluorescein angiography, late phase shows pinpoint hyperfluorescent hot spots. (K and $\mathbf{N}$ ) FAF images show a patchy pattern before treatment (K) and marked reduction of FAF 8 months after thermotherapy $(\mathbf{N})$. ( $(\mathbf{I}$ and $\mathbf{L})$ OCT scans demonstrate the elevated choroidal mass with subtle subretinal fluid. (L) Marked increased elevation of the tumor. (O) Disappearance of choroidal mass after treatment. The arrows at the color fundus photographs (A, G, J, M) correspond to the B-scan lines of the OCT images. Abbreviations: FAF, fundus autofluorescence; OCT, optical coherence tomography.

both patchy and diffuse FAF patterns. These FAF findings are almost identical to the FAF findings in patients with choroidal melanoma in the present study. Only a few authors have studied FAF overlying IML and found no characteristic patterns of FAF. ${ }^{8,10,23}$ Our study demonstrates that tumor characteristics, OCT, and FAF findings, were not valuable in differentiating an IML from choroidal melanoma or other choroidal tumors, but were valuable in monitoring tumor growth. Two of ten patients $(20 \%)$ with IML in this study showed tumor growth on follow-up $<12$ months. Tumor growth was only detected using OCT and FAF imaging. The presence of documented growth of an IML has been used as a surrogate for malignancy for many years and is important in identifying IMLs that require treatment. ${ }^{4,32-35}$ The two most important predictive clinical risk factors for tumor growth are the presence of SRF overlying the tumor and orange pigment 

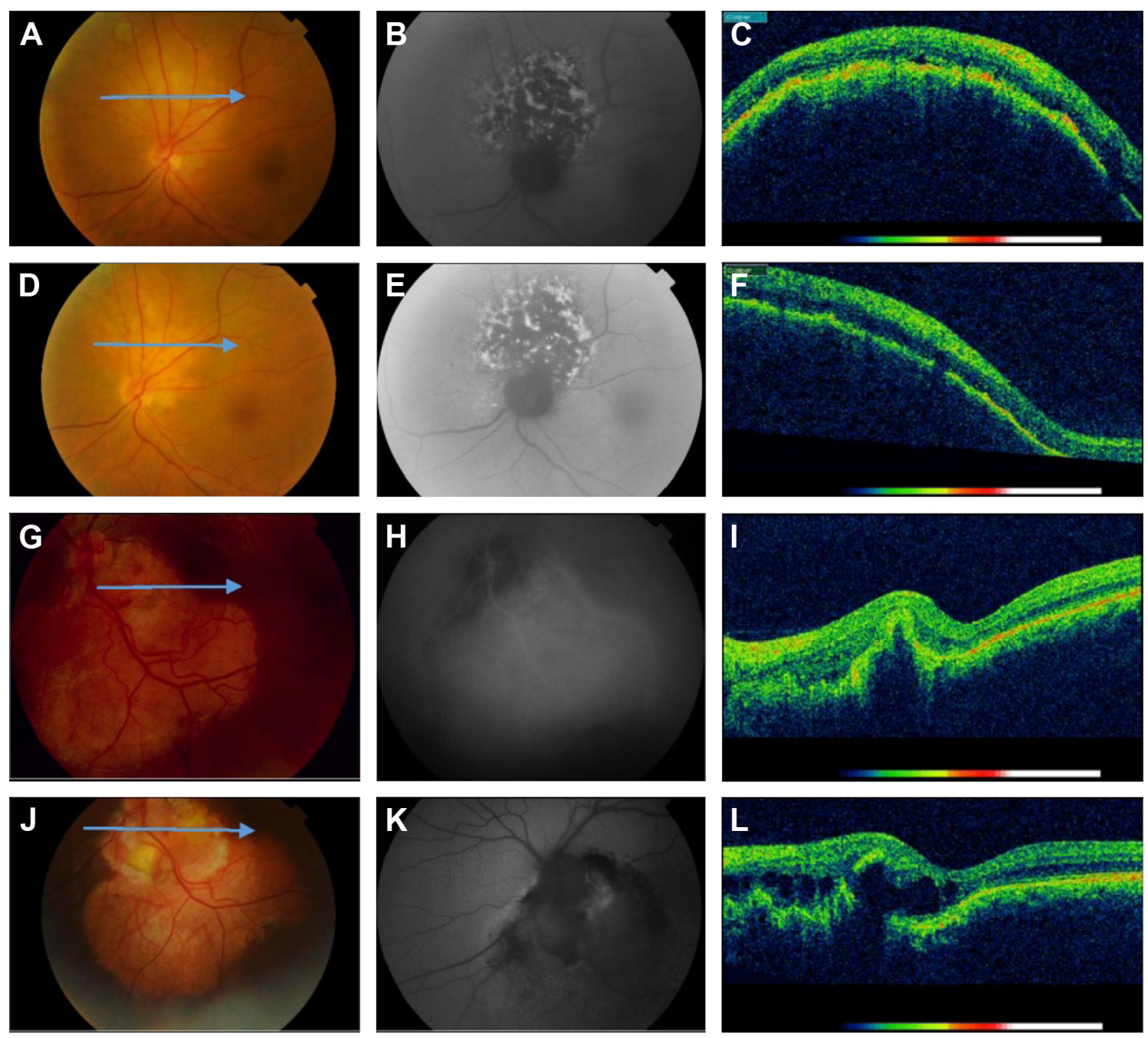

Figure 3 Color fundus photographs, FAF images, and OCT scans of patients with breast cancer metastasis and choroidal osteoma.

Notes: Images from a female aged 72 with breast cancer metastasis in her left eye (A-F): (A and $\mathbf{D})$ Color fundus photos show nonpigmented tumor (A) and 3 months after cytostatic agents choroidal mass reduction with partial pigmentation (D). (B and E) FAF images show a patchy pattern characterized by distinct areas of increased autofluorescence between areas of normal autofluorescence (B), and 3 months after treatment the FAF is unchanged (E). (C and $\mathbf{F})$ OCT scans before (C) and after treatment (F) show minimal intra- and subretinal fluid. Images from a female aged 30 with choroidal osteoma in her left eye (G-L). (G and J) Color fundus photos show orange-yellow inferotemporal tumor in contact with the optic nerve $(\mathbf{G})$ and minimal progress of the tumor 10 years later $(\mathbf{J})$. (H) Indocyanine green angiography, late phase shows diffuse hyperfluorescence due to staining. (K) FAF image shows mostly hypoautofluorescence due to RPE atrophy. (I and L) OCT scans show RPE detachment (I) and increased subretinal fluid 10 years later (L). The arrows at the color fundus photographs (A, D, G, J) correspond to the B-scan lines of the OCT images.

Abbreviations: FAF, fundus autofluorescence; OCT, optical coherence tomography; RPE, retinal pigment epithelium.

on the surface of the tumor. The evaluation of these two risk factors is most useful using OCT and FAF imaging. ${ }^{4,10}$

The choroidal melanomas in this study were nonpigmented in $40 \%$ of the cases, and $90 \%$ of the cases had SRF on OCT. Muscat et $\mathrm{al}^{7}$ detected SRF overlying the untreated choroidal melanomas in $100 \%$ of their cases using OCT. The FAF patterns of choroidal melanomas were classified as either patchy or diffuse in this study. This classification was used previously by Gündüz et al. ${ }^{8}$ They defined the patchy FAF pattern over choroidal melanomas as the presence of distinct areas of increased FAF between areas of normal autofluorescence, and the diffuse pattern of FAF was characterized by the presence of increased FAF with indistinct borders over a larger area of the tumor. We found that $60 \%$ of the cases of choroidal melanoma had a patchy FAF pattern, while Gündüz et $\mathrm{al}^{8}$ reported that $68 \%$ of choroidal melanoma cases had a diffuse FAF pattern. The higher rate of diffuse FAF patterns reported by Gündüz et $\mathrm{al}^{8}$ compared to the present results could be due to the fact that they included larger choroidal melanomas in their study, and their observations that the diffuse FAF pattern was significantly associated with increased tumor thickness and increased largest basal diameter. Shields et $\mathrm{al}^{27}$ also found that the hyperautofluorescence of choroidal melanomas increased with larger tumors, pigmented tumors, and those with disrupted overlying RPE. Only one of the ten $(10 \%)$ patients with choroidal melanoma in this study was 

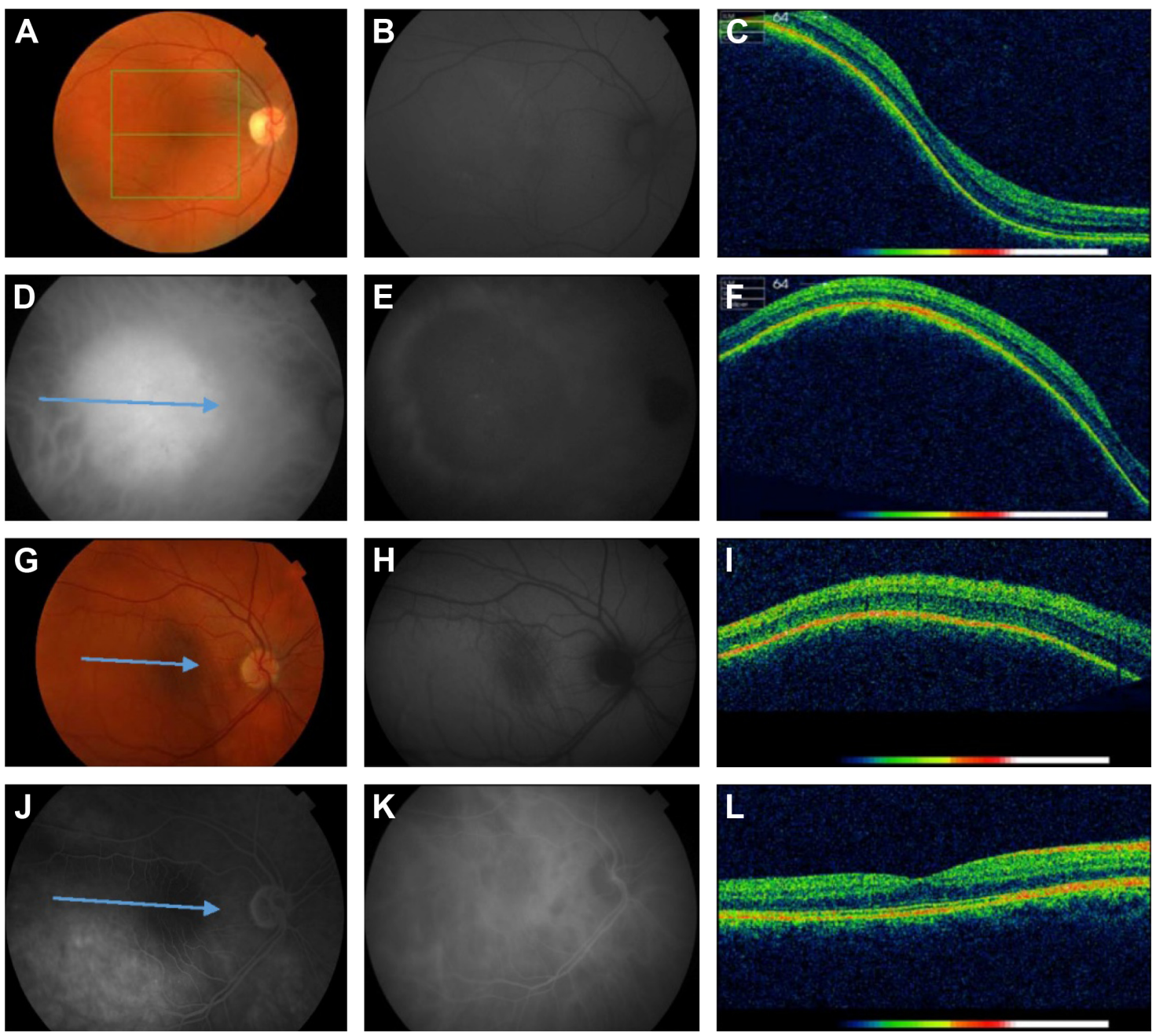

Figure 4 Color fundus photographs, FAF images, and OCT scans of patients with choroidal hemangioma and choroidal lymphoma metastasis.

Notes: Images from a female aged 42 with a choroidal hemangioma in her right eye (A-F). (A) Color fundus photo shows an orange-pink circumscribed tumor. (B) FAF image shows isoautofluorescence over the tumor. (C and F) OCT scans of the tumor reveal the abruptly elevated choroidal mass without intra- or subretinal fluid. (D and E) ICG in the midphase shows homogeneous hyperfluorescence of the tumor (D) and in the late phase dye washout with peripheral leakage (E). Images from a female aged 34 with a choroidal metastasis of systemic lymphoma in her right eye (G-L). (G) Color fundus photo shows a nonpigmented choroidal mass. (H) FAF image shows isoautofluorescence of the tumor. (I) OCT scan reveals the abruptly elevated choroidal mass. (J) FA, late phase shows minimal hyperfluorescence. (K) ICG angiography, late phase shows hypoautofluorescence of the tumor. (L) Normalized OCT scan I month after treatment with cytostatic agents. The arrows at ICG image (D), the color fundus photograph (G), and FA image (J) correspond to the B-scan lines of the OCT images.

Abbreviations: FA, fluorescein angiography; FAF, fundus autofluorescence; ICG, indocyanine green angiography; OCT, optical coherence tomography.

diagnosed by transretinal biopsy. The majority of patients with malignant choroidal melanoma are today managed by brachytherapy. This is often done without biopsy for histopathological, molecular, or cytological analysis, which prevents adequate prognosis based on histopathological and genetic analysis. Kivelä and Kujala ${ }^{43}$ reported that the most adequate criteria for prognosis of choroidal melanomas were analysis of chromosomal alternations and gene expression profiling. The presence of only one copy of chromosome 3 (complete monosomy 3) was observed in many choroidal melanomas that had metastasized and at 3-year follow-up, for tumors with complete monosomy 3, the occurrence of metastasis was $0 \%$ for small, $24.4 \%$ for medium, and $57.5 \%$ for large melanomas. ${ }^{41,43}$ The recent findings by Shields et $\mathrm{al}^{37}$ that each extra millimeter increase in melanoma thickness was associated with $5 \%$ increased risk of metastasis and Damato et $\mathrm{al}^{38}$ that ocular treatment of choroidal melanoma may prevent tumor growth, dedifferentiation, and metastatic disease in younger patients with small and less malignant tumors indicate the importance of early and accurate diagnosis by sound clinical evaluation, including OCT, FAF imaging, and biopsy.

Patients with other choroidal tumors in this study exhibited no significant difference regarding clinical and tumor characteristics compared with IMLs and choroidal melanoma cases. All were nonpigmented, and only $30 \%$ of the cases had 
SRF on OCT. None of our cases of choroidal hemangioma showed SRF on OCT. A low rate of the SRF on OCT (in only $19 \%$ of the cases with choroidal hemangioma) was also reported by Ramasubramanian et al. ${ }^{31}$ We found SRF on OCT in $67 \%$ of the cases with osteoma (all had choroidal neovascularization), while Freton and Finger ${ }^{16}$ found SRF in only $27 \%$ of the cases with osteoma. Our patients with choroidal metastatic lesions exhibited SRF in $50 \%$ of cases, whereas Collet et $\mathrm{al}^{24}$ found SRF in $73 \%$ of their cases. In two of our cases (breast cancer metastasis and choroidal lymphoma metastasis), OCT was valuable in monitoring the response to treatment (Figures 3 and 4). OCT and FAF imaging have been demonstrated to be important for monitoring the response to treatment of intraocular tumors by several authors. ${ }^{20}$ No characteristic patterns of FAF were found in our patients with other choroidal tumors.

Previous studies on FAF imaging in intraocular tumors have revealed that choroidal nevi typically had overlying hypoautofluorescence due to the degeneration or atrophy of RPE cells, and choroidal melanomas, especially large melanomas, generally had confluent/diffuse overlying hyperfluorescence due to the accumulation of lipofuscin within the RPE cells and within SRF. ${ }^{8-10,22,27,30}$ Patients with choroidal metastases and choroidal hemangiomas in the present study showed both a patchy FAF pattern and isoautofluorescence. It has been reported that choroidal metastases and choroidal hemangiomas often exhibit overlying hyperautofluorescence that is correlated to focal lipofuscin accumulation in the overlying RPE cells as well as in SRF, particularly in acute tumors. ${ }^{24,30,31}$ Two patients with osteoma in this study had both hypo- and hyperautofluorescence (mixed) overlying the tumors. Characteristic FAF patterns have recently been reported in patients with osteomas, with isoautofluorescence overlying calcified regions of the tumor due to the intact RPE, and general hypoautofluorescence in decalcified regions due to hyperplasia, metaplasia, and atrophy of the RPE. ${ }^{25,30}$

Our study had several limitations, including the small patient number in each studied group, and the grading of patchy versus diffuse FAF pattern of intraocular tumors was not done in a masked fashion but interobserver variability was tested in the classification of FAF pattern. All patients but one had the preliminary diagnosis based on clinical tumor features, funduscopy, ultrasound measurements, and FA/ICG findings.

There is thus considerable evidence that OCT and FAF imaging should be incorporated into diagnostic algorithms for intraocular tumors in clinical care in order to improve the characterization of tumors, and promote early diagnosis and treatment to improve patient survival and decrease the risk of metastasis, especially in patients with IML. ${ }^{4,6-8,10,11,20,22-31}$

\section{Conclusion}

This study demonstrates that both OCT and FAF were helpful in the differential diagnosis of choroidal nevi versus IMLs, choroidal melanomas, and other choroidal tumors. The accurate and periodical clinical evaluation of patients with intraocular tumors using ultrasound in combination with OCT and FAF for the detection of both SRF and characteristic FAF patterns overlying the tumors may be useful for detection of tumor growth.

\section{Acknowledgments}

This study was supported by Stiftelsen för synskadade I fd Malmöhus län and Skane University Hospital.

\section{Disclosure}

The authors report no conflicts of interest in this work.

\section{References}

1. Heimann H, Jmor F, Damato B. Imaging of retinal and choroidal vascular tumours. Eye. 2013;27(2):208-216.

2. Schmitz-Valckenberg S, Holz FG, Bird AC, Spaide RF. Fundus autofluorescence imaging. Review and perspectives. Retina. 2008;28(3): 385-409.

3. Spaide R. Autofluorescence from the outer retina and subretinal space, hypothesis and review. Retina. 2008;28(1):5-35.

4. Espinoza G, Rosenblatt B, Harbour JW. Optical coherence tomography in the evaluation of retinal changes associated with suspicious choroidal melanocytic tumors. Am J Ophthalmol. 2004;137(1):90-95.

5. Schlitt MA, Kaliki S, Shields CL. Reduction of orange pigment overlying choroidal melanoma following plaque radiotherapy. Retina Today. 2013;(9):35-36.

6. Say EA, Shah SU, Ferenczy S, Shields CL. Optical coherence tomography of retinal and choroidal tumors. J Ophthalmol. 2011;2011: 385058.

7. Muscat S, Parks S, Kemp E, Keating D. Secondary retinal changes associated with choroidal naevi and melanomas documented by optical coherence tomography. Br J Ophthalmol. 2004;88(1):120-124.

8. Gündüz K, Pulido JS, Pulido JE, Link T. Correlation of fundus autofluorescence with fluorescein and indocyanine green angiography in choroidal melanocytic lesions. Retina. 2008;28(9):1257-1264.

9. Shields CL, Bianciotto C, Pirondini C, Materin MA, Harmon SA, Shields JA. Autofluorescence of orange pigment overlying small choroidal melanoma. Retina. 2007;27(8):1107-1111.

10. Singh A, Belfort RN, Sayanagi K, Kaiser PK. Fourier domain optical coherence tomographic and auto-fluorescence findings in indeterminate choroidal melanocytic lesions. Br J Ophthalmol. 2010;94(4): 474-478.

11. Materin MA, Raducu R, Bianciotto C, Shields CL. Fundus autofluorescence and optical coherence tomography findings in choroidal melanocytic lesions. Middle East Afr J Ophthalmol. 2010;17(3):201-206.

12. Wojtkowski M, Bajraszewski T, Gorczyńska I, et al. Ophthalmic imaging by spectral optical coherence tomography. Am J Ophthalmol. 2004;138(3):412-419.

13. Shields CL, Mashayekhi A, Materin MA, et al. Optical coherence tomography of choroidal nevus in 120 patients. Retina. 2005;25(3): 243-252. 
14. Shah SU, Kaliki S, Shields CL, Ferenczy SR, Harmon SA, Shields JA. Enhanced depth imaging optical coherence tomography of choroidal nevus in 104 cases. Ophthalmology. 2012;119(5):1066-1072.

15. Shields CL, Kaliki S, Rojanaporn D, Ferenczy S, Shields JA. Enhanced depth imaging optical coherence tomography of small choroidal melanoma. Arch Ophthalmol. 2012;130(7):850-856.

16. Freton A, Finger PT. Spectral domain-optical coherence tomography analysis of choroidal osteoma. Br J Ophthalmol. 2012;96(2):224-228.

17. Egawa M, Mitamura Y, Hayashi Y, Naito T. Spectral-domain optical coherence tomographic and fundus autofluorescence findings in eyes with primary intraocular lymphoma. Clin Ophthalmol. 2014;8:335-341.

18. Egawa M, Mitamura Y, Hayashi Y, Semba K, Naito T. Changes of fundus autofluorescence and spectral-domain optical coherence tomographic findings after treatment of primary intraocular lymphoma. J Ophthalmic Inflamm Infect. 2014;4(1):7.

19. Delori FC, Dorey K, Staurenghi G, Arend O, Goger DG, Weiter JJ. In vivo fluorescence of the ocular fundus exhibits retinal pigment epithelium lipofuscin characteristics. Invest Ophthalmol Vis Sci. 1995;36(3): 718-729.

20. Nandakumar N, Buzney S, Weiter JJ. Lipofuscin and the principles of fundus autofluorescence: a review. Semin Ophthalmol. 2012;27(5-6): 197-201.

21. Nilsson SE, Sundelin SP, Wihlmark U, Brunk UT. Aging of cultured retinal pigment epithelial cells: oxidative reactions, lipofuscin formation and blue light damage. Doc Ophthalmol. 2003;106(1):13-16.

22. Gündüz K, Pulido JS, Bakri SJ, Petit-Fond E. Fundus autofluorescence in choroidal melanocytic lesions. Retina. 2007;27(6):681-687.

23. Lavinsky D, Belfort RN, Navajas E, Torres V, Martins MC, Belfort R Jr. Fundus autofluorescence of choroidal nevus and melanoma. $\mathrm{Br} \mathrm{J}$ Ophthalmol. 2007;91(10):1299-1302.

24. Collet LC, Pulido JS, Gündüz K, et al. Fundus autofluorescence in choroidal metastatic lesions. A pilot study. Retina. 2008;28(9): 1251-1256.

25. Sisk RA, Riemann CD, Petersen MR, et al. Fundus autofluorescence findings of choroidal osteoma. Retina. 2013;33(1):97-104.

26. Shields CL, Pirondini C, Bianciotto C, Materin MA, Harmon SA, Shields JA. Autofluorescence of choroidal nevus in 64 cases. Retina. 2008;28(8):1035-1043.

27. Shields CL, Bianciotto C, Pirondini C, Materin MA, Harmon SA, Shields JA. Autofluorescence of choroidal melanoma in 51 cases. $\mathrm{Br} J$ Ophthalmol. 2008;92(5):617-622.

28. Ishida T, Ohno-Matsui K, Kaneko Y, et al. Fundus autofluorescence patterns in eyes with primary intraocular lymphoma. Retina. 2010;30(1): $23-32$.
29. Casady M, Faia L, Nazemzadeh M, Nussenblatt R, Chan CC, Sen HN. Fundus autofluorescence patterns in primary intraocular lymphoma. Retina. 2014;34(2):366-372.

30. Almeida A, Kaliki S, Shields CL. Autofluorescence of intraocular tumours. Curr Opin Ophthalmol. 2013;24(3):222-232.

31. Ramasubramanian A, Shields Cl, Harmon SA, Shields JA. Autofluorescence of choroidal hemangioma in 34 consecutive eyes. Retina. 2010;30(1):16-22.

32. Shields CL, Shields JA, Kiratli H, De Potter P, Cater JR. Risk factors for growth and metastasis of small choroidal melanocytic lesions. Ophthalmology. 1995;102(9):1351-1361.

33. COMS Report no 5. Factors predictive of growth and treatment of small choroidal melanoma. Arch Ophthalmol. 1997;115(12):1537-1544.

34. Shields CL, Cater J, Shields JA, Singh AD, Santos MCM, Carvalho C. Combination of clinical factors predictive of growth of small choroidal melanocytic tumors. Arch Ophthalmol. 2000;118(3):360-364.

35. Rishi P, Koundanya VV, Shields CL. Using risk factors for detection and prognostication of uveal melanoma. Indian J Ophthalmol. 2015; 63(2):110-116.

36. Singh AD, Turell ME, Topham AK. Uveal melanoma: Trends in incidence, treatment, and survival. Ophthalmology. 2011;118(9):1881-1885.

37. Shields CL, Furuta M, Thangappan A, et al. Metastasis of uveal melanoma millimeter-by-millimeter in 8033 consecutive eyes. Arch Ophthalmol. 2009;127(8):989-998.

38. Damato BE, Heimann H, Kalirai H, Coupland SE. Age, survival predictors, and metastatic death in patients with choroidal melanoma. JAMA Ophthalmol. 2014;132(5):605-613.

39. Shields CL, Ganguly A, Materin MA, et al. Chromosome 3 analysis of uveal melanoma using fine-needle aspiration biopsy at the time of plaque radiotherapy in 140 consecutive cases. Trans Am Ophthalmol Soc. 2007;105:43-53.

40. Van Raamsdonk CD, Griewank KG, Crosby MB, et al. Mutations in GNA 11 in uveal melanoma. $N$ Engl J Med. 2010;363(23):2191-2199.

41. Shields CL, Ganguly A, Bianciotto CG, Turaka K, Tavallali A, Shields JA. Prognosis of uveal melanoma in 500 cases using genetic testing of fine-needle aspiration biopsy specimens. Ophthalmology. 2011;118(2):396-401.

42. Finger PT, Chin KJ, Semenova EA. Intravitreal anti-VEGF therapy for macular radiation retinopathy: a 10-year study. Eur J Ophthalmol. 2016;26(1):60-66.

43. Kivelä T, Kujala E. Prognostication in eye cancer: the latest tumor, node, metastasis classification and beyond. Eye. 2013;27(2):243-252.
Clinical Ophthalmology

\section{Publish your work in this journal}

Clinical Ophthalmology is an international, peer-reviewed journal covering all subspecialties within ophthalmology. Key topics include: Optometry; Visual science; Pharmacology and drug therapy in eye diseases; Basic Sciences; Primary and Secondary eye care; Patient Safety and Quality of Care Improvements. This journal is indexed on Submit your manuscript here: http://www.dovepress.com/clinical-ophthalmology-journal
Dovepress

PubMed Central and CAS, and is the official journal of The Society of Clinical Ophthalmology (SCO). The manuscript management system is completely online and includes a very quick and fair peer-review system, which is all easy to use. Visit http://www.dovepress.com/ testimonials.php to read real quotes from published authors. 